\title{
Moderate Laws of Large Numbers via Weak Laws
}

\author{
Yu-Lin Chou*
}

\begin{abstract}
By a moderate law of large numbers we mean any theorem whose conclusion includes the $L^{p}$-vanishment of the sequence of the sample means of some centered random variables with $1 \leq p<+\infty$ given. Given any $1 \leq p<+\infty$ and any $\varepsilon>0$, we prove a moderate law of large numbers for $L^{p+\varepsilon}$-bounded random variables that obey a weak law. Thus our moderate laws in particular complement those obtained from the martingale theory, and establish the counterintuitive fact that (for $L^{p+\varepsilon}$-bounded random variables) where there is a weak law there is a moderate law.
\end{abstract}

Keywords: $L^{p}$-boundedness; $L^{p}$-convergence of sample means; moderate laws of large numbers; weak laws of large numbers

MSC 2020: 60F 25; 60F05; 28A20

\section{Introduction}

For communication purposes, we will refer to any law of large numbers whose concerned mode of convergence is $L^{p}$-convergence as a moderate law of large numbers (MLLN). The modifier "moderate" is adopted with respect to the connections between $L^{p}$-convergence, almost everywhere convergence, and convergence in measure, and is certainly meant to echo its presence in the established term "moderate deviation principle" in probability theory.

The martingale theory furnishes some basic MLLNs.

We wish to add that, given any $1 \leq p<+\infty$ and any $\varepsilon>0$, an $L^{p+\varepsilon}$-bounded sequence of random variables $X_{1}, X_{2}, \ldots$ obeying a weak law, i.e. such that $n^{-1} \sum_{i=1}^{n}\left(X_{i}-\right.$ $\left.\mathbb{E} X_{i}\right) \rightsquigarrow 0$, turns out to obey an MLLN; this holds without imposing any dependence structure nor any further distributional homogeneity requirement.

Evidently, we then in general have MLLNs directly from the established laws of large numbers, strong or weak. Moreover, our MLLNs take care of the cases where the martingale theory need not apply.

${ }^{*}$ Yu-Lin Chou, Institute of Statistics, National Tsing Hua University, Hsinchu 30013, Taiwan, R.O.C.; Email: y.1.chou@gapp.nthu.edu.tw 


\section{Results}

For all $1 \leq p<+\infty$, by $L^{p}$-boundedness of a sequence of $L^{p}$ real-valued functions we mean the boundedness of the sequence with respect to the $L^{p}$-norm.

We begin by proving our MLLNs:

Theorem 1. Let $1 \leq p<+\infty$; let $\Omega$ be a probability space with $\mathbb{P}$ denoting the given probability measure. If $X_{1}, X_{2}, \ldots$ are random variables obeying a weak law, and if there is some $\varepsilon>0$ such that $X_{1}, X_{2}, \ldots$ are $L^{p+\varepsilon}$-bounded, then

$$
\left|n^{-1} \sum_{i=1}^{n}\left(X_{i}-\mathbb{E} X_{i}\right)\right|_{L^{p}} \rightarrow 0 \quad \text { as } n \rightarrow \infty .
$$

Proof. There is by assumption some real $M>0$ such that $\sup _{i \in \mathbb{N}}\left|X_{i}\right|_{L^{p+\varepsilon}} \leq M / 2$. If

$$
Y_{n}:=n^{-1} \sum_{i=1}^{n}\left(X_{i}-\mathbb{E} X_{i}\right)
$$

for all $n \in \mathbb{N}$, then Minkowski's and Jensen's inequalities jointly imply

$$
\begin{aligned}
\left|Y_{n}\right|_{L^{p+\varepsilon}} & \leq n^{-1} \sum_{i=1}^{n}\left|X_{i}-\mathbb{E} X_{i}\right|_{L^{p+\varepsilon}} \\
& \leq n^{-1} \sum_{i=1}^{n}\left|X_{i}\right|_{L^{p+\varepsilon}}+n^{-1} \sum_{i=1}^{n}\left|\mathbb{E} X_{i}\right|_{L^{p+\varepsilon}} \\
& \leq M / 2+n^{-1} \sum_{i=1}^{n}\left|X_{i}\right|_{L^{1}} \\
& \leq M
\end{aligned}
$$

for all $n \in \mathbb{N}$.

Given any subsequence $\left(Y_{n_{j}}\right)_{j \in \mathbb{N}}$ of $\left(Y_{n}\right)_{n \in \mathbb{N}}$, there is by the assumed weak law abidance some further subsequence $\left(Y_{n_{j(k)}}\right)_{k \in \mathbb{N}}$ such that $\left|Y_{n_{j(k)}}\right|^{p} \rightarrow_{a . s .} 0$ as $k \rightarrow \infty$. Since $\left(Y_{n_{j(k)}}\right)_{k \in \mathbb{N}}$ is $L^{p+\varepsilon}$-bounded, the sequence $\left(\left|Y_{n_{j(k)}}\right|^{p}\right)_{k \in \mathbb{N}}$ is uniformly integrable. It then follows by Vitali convergence theorem (Rudin [2], for concreteness) that

$$
\mathbb{E}\left|Y_{n_{j(k)}}\right|^{p} \rightarrow 0 \text { as } k \rightarrow \infty
$$

Now since $\mathbb{E}\left|Y_{n}\right|^{p} \rightarrow 0$ as $n \rightarrow \infty$, we have

$$
\left|Y_{n}\right|_{L^{p}} \rightarrow 0 \text { as } n \rightarrow \infty
$$

this completes the proof.

It follows immediately from Theorem 1 that MLLNs may in many cases, including those not covered by the martingale theory, be obtained as corollaries to the known laws of large numbers. For instance, Theorem 1 and Etemadi's strong law (Etemadi [1]) jointly imply 
Corollary 1. Let $\Omega$ be a probability space with $\mathbb{P}$ denoting the given probability measure. If $X_{1}, X_{2}, \ldots$ are independent (pairwise or not) identically distributed random variables, and if there is some $\varepsilon>0$ such that $X_{1} \in L^{1+\varepsilon}(\mathbb{P})$, then

$$
\left|n^{-1} \sum_{i=1}^{n}\left(X_{i}-\mathbb{E} X_{i}\right)\right|_{L^{1}} \rightarrow 0 \text { as } n \rightarrow \infty .
$$

\section{References}

[1] Etemadi, N. (1981). An elementary proof of the strong law of large numbers. $Z$. Wahrscheinlichkeitstheorie verw. Gebiete 55 119-122.

[2] Rudin, W. (1987). Real and Complex Analysis, (international) third edition. McGraw-Hill. 